\title{
Supplementary Information Better Informed Distance Geometry: Using What We Know to Improve Conformation Generation
}

\author{
Sereina Riniker, ${ }^{*} \dagger$ and Gregory A. Landrum ${ }^{\ddagger}$ \\ †Laboratory of Physical Chemistry, ETH Zürich, Vladimir-Prelog-Weg 2, 8093 Zürich, \\ Switzerland \\ $\ddagger$ Novartis Institutes for BioMedical Research, Novartis Pharma AG, Novartis Campus, \\ 4056 Basel, Switzerland \\ E-mail: sriniker@ethz.ch
}

Figure S1: Comparison of the final torsional potentials (red lines) for 387 patterns in the range $\left[0^{\circ}-360^{\circ}\right]$ with the normed torsional-angle distributions from CSD used for fitting.

Figure S2: Comparison of the final torsional potentials (red lines) for 374 patterns in the range $\left[0^{\circ}-360^{\circ}\right]$ with the torsion ranges from Ref. $\left[^{1}\right]$ (purple rectangles for tolerance 1 , light purple rectangles for tolerance 2 , dark purple lines for the peaks).

Figure S3: Comparison of the final torsional potentials (red lines) for 302 patterns in the range $\left[0^{\circ}-360^{\circ}\right]$ with the normed torsional-angle distributions in generated conformers from DG for the combined data sets (1959 molecules). 
Figure S4: Comparison of the final torsional potentials (red lines) for 302 patterns in the range $\left[0^{\circ}-360^{\circ}\right]$ with the normed torsional-angle distributions in generated conformers from ETDG for the combined data sets (1959 molecules).

Figure S5: Comparison of the final torsional potentials (red lines) for 302 patterns in the range $\left[0^{\circ}-360^{\circ}\right]$ with the normed torsional-angle distributions in generated conformers from ETKDG for the combined data sets (1959 molecules).
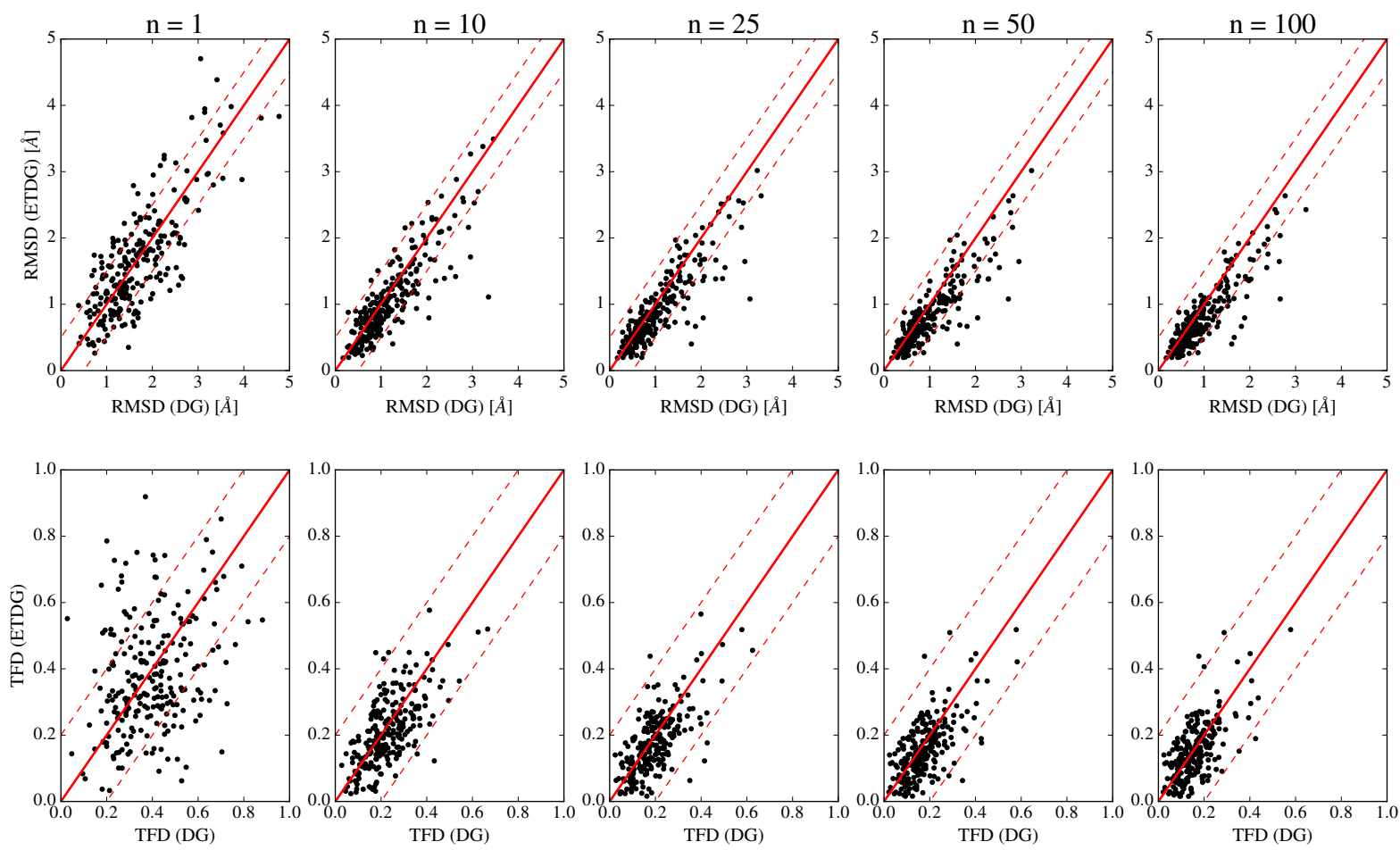

Figure S6: Comparison of the best RMSD (top) and best TFD (bottom) values (reference is crystal conformation) of DG and ETDG as a function of number of conformers considered, $n$, for the PDB data set (238 molecules). 

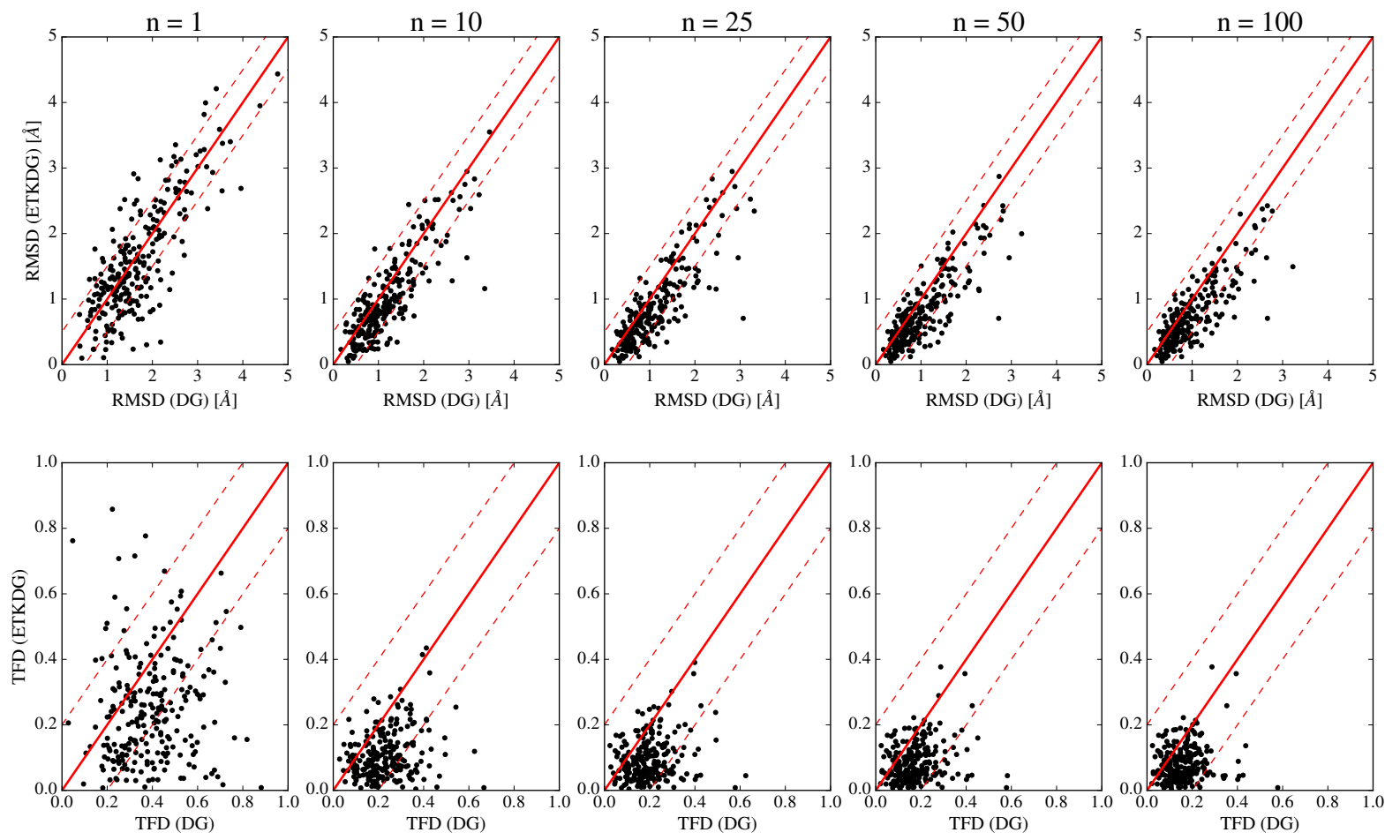

Figure S7: Comparison of the best RMSD (top) and best TFD (bottom) values (reference is crystal conformation) of DG and ETKDG as a function of number of conformers considered, $n$, for the PDB data set (238 molecules). 

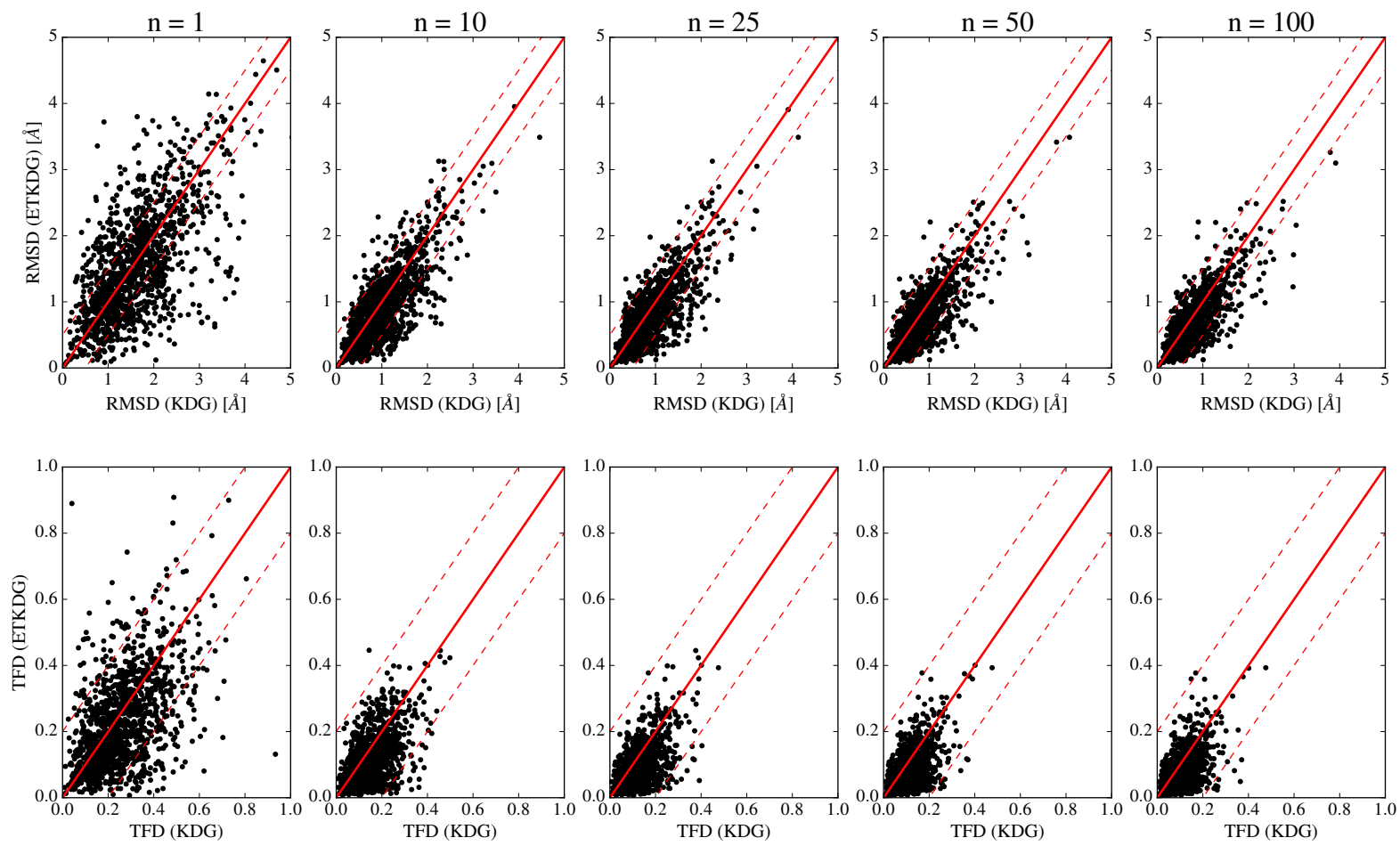

Figure S8: Comparison of the best RMSD (top) and best TFD (bottom) values (reference is crystal conformation) of KDG and ETKDG as a function of number of conformers considered, $n$, for the CSD data set (1290 molecules). 

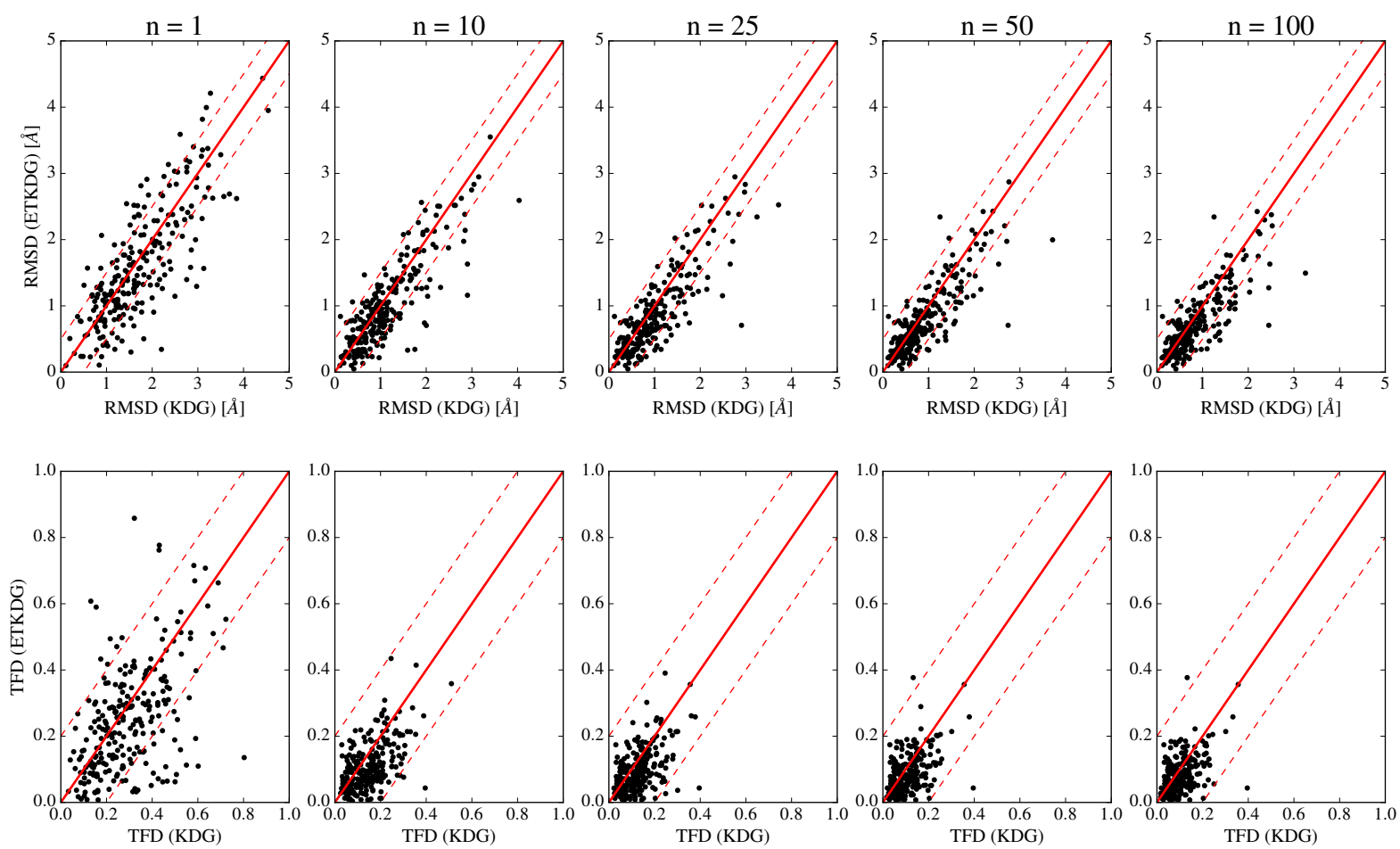

Figure S9: Comparison of the best RMSD (top) and best TFD (bottom) values (reference is crystal conformation) of KDG and ETKDG as a function of number of conformers considered, $n$, for the PDB data set (238 molecules).
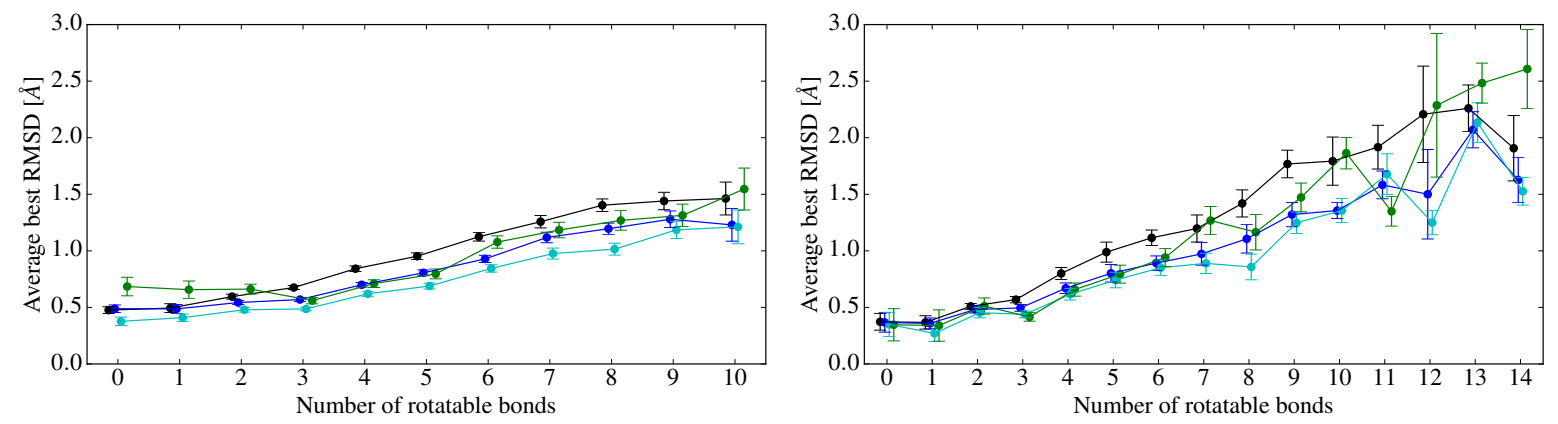

Figure S10: Average best RMSD as a function of the number of rotatable bonds using the CSD data set (left, 1290 molecules) and the PDB data set (right, 238 molecules) for $n=$ 100. The error bars represent the standard error. The data points are hittered horizontally to avoid overplotting. 

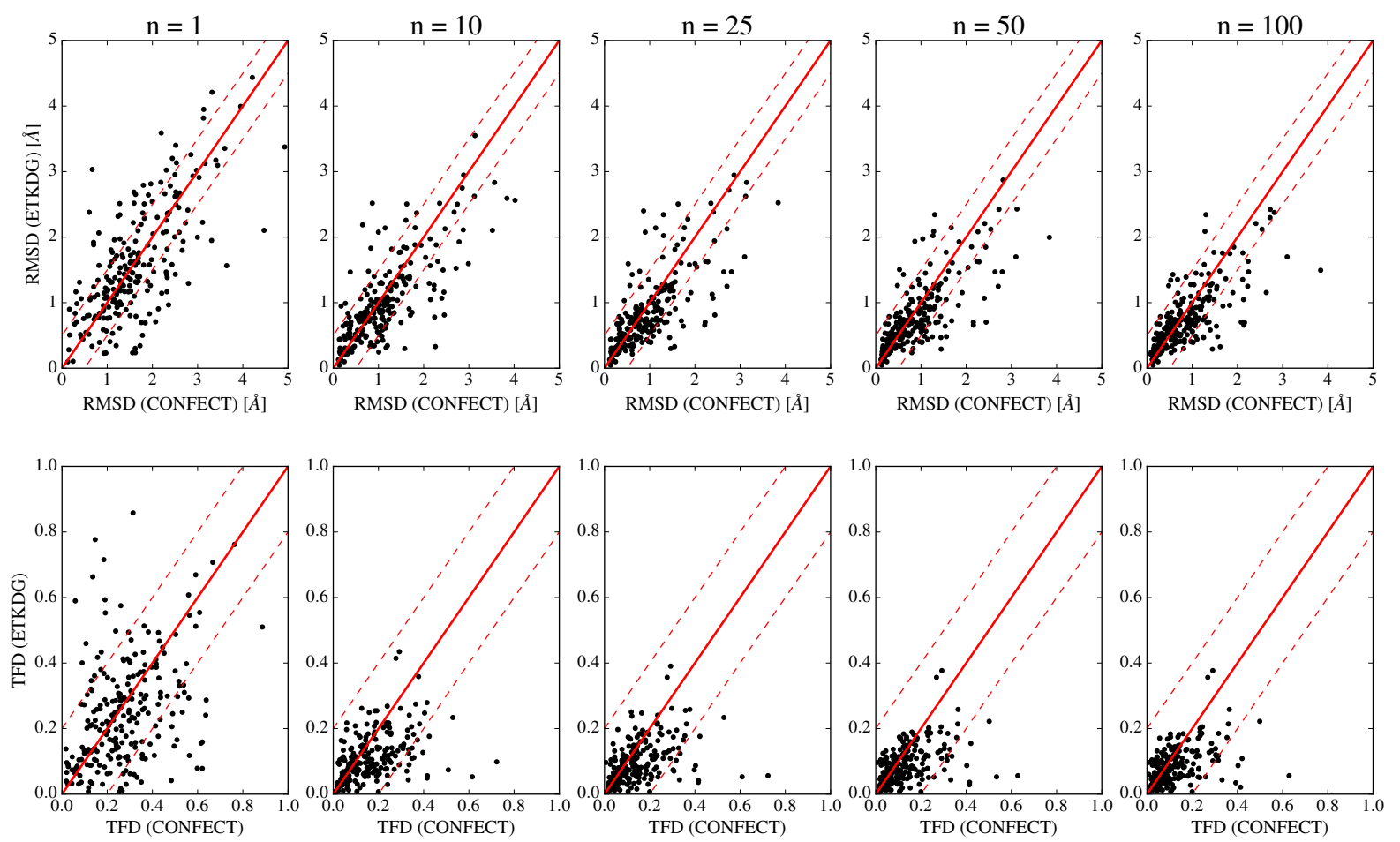

Figure S11: Comparison of the best RMSD (top) and TFD (bottom) to the crystal structure between CONFECT and ETKDG (bottom) as a function of number of conformers considered, $n$, for the PDB data set (235 molecules). 

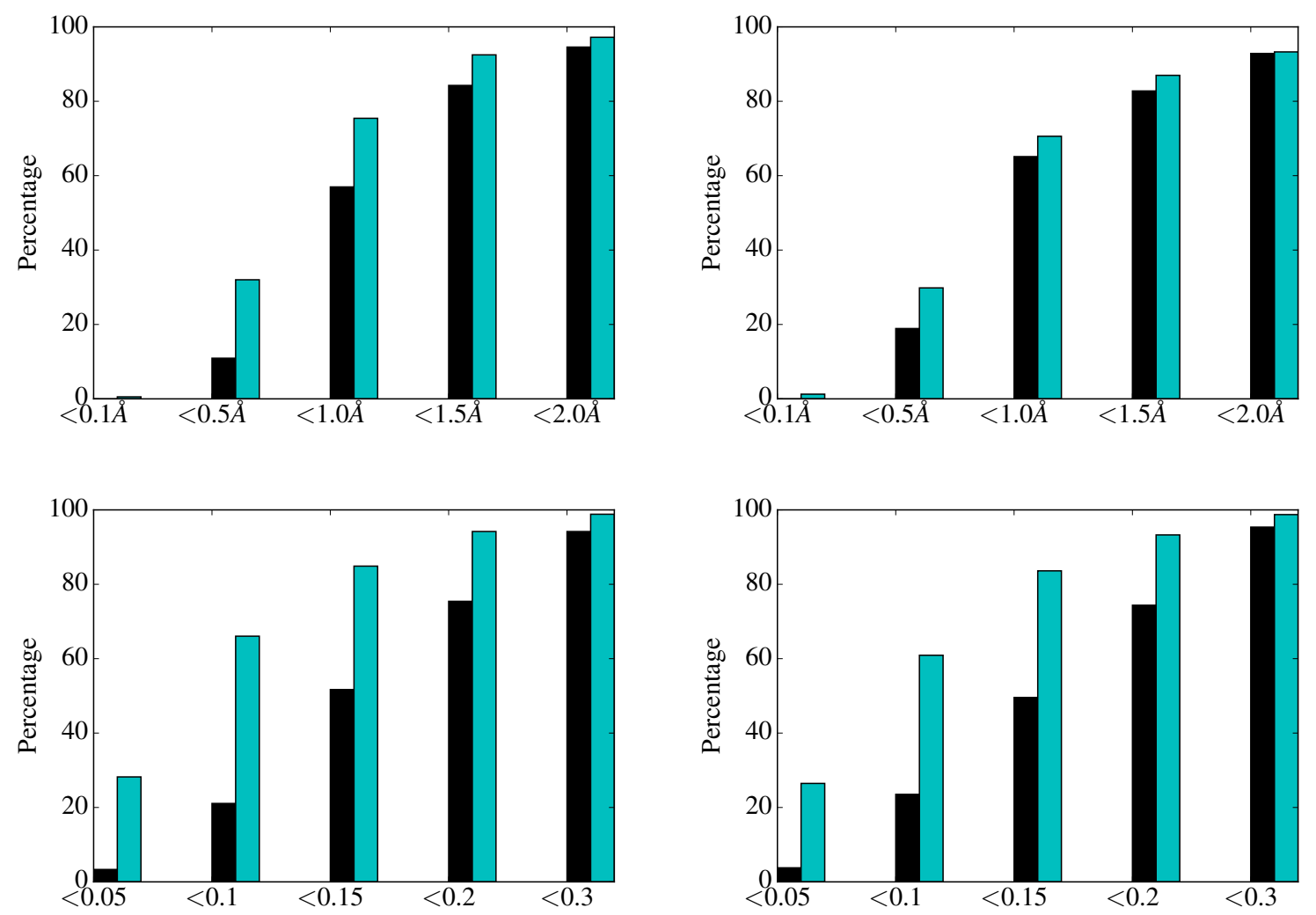

Figure S12: Percentage of molecules where the crystal conformation was reproduced within a certain RMSD cutoff $(0.1,0.5,1.0,1.5$ and $2.0 \AA)$ or TFD cutoff $(0.01,0.05,0.1,0.2,0.3)$ using the CSD data set (left, 1290 molecules) and the PDB data set (right, 238 molecules). DG $(n=100)$ is shown in black bars and ETKDG $(n=25)$ in cyan.

Table S1: $p$-values from one-sided paired $t$-tests of RMSD and TFD values of DG, KDG, and ETKDG for different number of conformers, $n$, using the CSD and the PDB data sets. The null hypothesis states that there is no difference between the RMSD values $\left(\mu=\mu_{0}=\right.$ $0)$, the alternative hypothesis is that the difference is greater than zero $\left(\mu>\mu_{0}\right)$, i.e. the second method is performing better than the first method. If the $p$-value is smaller than 0.05, the null hypothesis is discarded. Cases where the null hypothesis cannot be discarded are underlined.

\begin{tabular}{lll|rrrrr}
\hline Methods & Set & Measure & $n=1$ & $n=10$ & $n=25$ & $n=50$ & $n=100$ \\
\hline DG-KDG & CSD & RMSD & $1.2 \cdot 10^{-9}$ & $<2.2 \cdot 10^{-16}$ & $<2.2 \cdot 10^{-16}$ & $<2.2 \cdot 10^{-16}$ & $<2.2 \cdot 10^{-16}$ \\
& & TFD & $<2.2 \cdot 10^{-16}$ & $<2.2 \cdot 10^{-16}$ & $<2.2 \cdot 10^{-16}$ & $<2.2 \cdot 10^{-16}$ & $<2.2 \cdot 10^{-16}$ \\
& PDB & RMSD & $7.1 \cdot 10^{-1}$ & $5.4 \cdot 10^{-5}$ & $6.7 \cdot 10^{-9}$ & $1.6 \cdot 10^{-12}$ & $1.1 \cdot 10^{-14}$ \\
& & TFD & $4.0 \cdot 10^{-14}$ & $<2.2 \cdot 10^{-16}$ & $<2.2 \cdot 10^{-16}$ & $<2.2 \cdot 10^{-16}$ & $<2.2 \cdot 10^{-16}$ \\
\hline KDG-ETKDG & CSD & RMSD & $3.2 \cdot 10^{-3}$ & $<2.2 \cdot 10^{-16}$ & $<2.2 \cdot 10^{-16}$ & $<2.2 \cdot 10^{-16}$ & $<2.2 \cdot 10^{-16}$ \\
& & TFD & $7.8 \cdot 10^{-15}$ & $<2.2 \cdot 10^{-16}$ & $<2.2 \cdot 10^{-16}$ & $<2.2 \cdot 10^{-16}$ & $<2.2 \cdot 10^{-16}$ \\
& \multirow{2}{*}{ PDB } & RMSD & $2.5 \cdot 10^{-3}$ & $1.9 \cdot 10^{-7}$ & $8.6 \cdot 10^{-11}$ & $5.7 \cdot 10^{-9}$ & $3.1 \cdot 10^{-9}$ \\
& & TFD & $3.9 \cdot 10^{-6}$ & $<2.2 \cdot 10^{-16}$ & $2.2 \cdot 10^{-13}$ & $1.3 \cdot 10^{-9}$ & $2.7 \cdot 10^{-8}$ \\
\hline
\end{tabular}


Table S2: $p$-values from one-sided paired $t$-tests of RMSD and TFD values of CONFECT, and ETKDG for different number of conformers, $n$, using the CSD and the PDB data sets. The null hypothesis states that there is no difference between the RMSD values ( $\mu=\mu_{0}=$ $0)$, the alternative hypothesis is that the difference is greater than zero $\left(\mu>\mu_{0}\right)$, i.e. the second method is performing better than the first method. If the $p$-value is smaller than 0.05, the null hypothesis is discarded. Cases where the null hypothesis cannot be discarded are underlined.

\begin{tabular}{lll|rrrrr}
\hline Methods & Set & Measure & $n=1$ & $n=10$ & $n=25$ & $n=50$ & $n=100$ \\
\hline CONFECT-ETKDG & CSD & RMSD & $\underline{1.00}$ & $3.6 \cdot 10^{-3}$ & $5.9 \cdot 10^{-6}$ & $1.0 \cdot 10^{-8}$ & $5.7 \cdot 10^{-10}$ \\
& & TFD & $\underline{1.00}$ & $2.9 \cdot 10^{-13}$ & $<2.2 \cdot 10^{-16}$ & $<2.2 \cdot 10^{-16}$ & $<2.2 \cdot 10^{-16}$ \\
& PDB & RMSD & $\underline{0.50}$ & $7.8 \cdot 10^{-3}$ & $1.3 \cdot 10^{-3}$ & $1.6 \cdot 10^{-3}$ & $8.0 \cdot 10^{-4}$ \\
& & TFD & $3.0 \cdot 10^{-2}$ & $5.0 \cdot 10^{-11}$ & $5.2 \cdot 10^{-10}$ & $1.4 \cdot 10^{-9}$ & $1.8 \cdot 10^{-8}$ \\
\hline
\end{tabular}

Table S3: $p$-values from one-sided paired $t$-tests of RMSD and TFD values of DG, KDG, and ETKDG for different number of conformers, $n$, using the CSD and the PDB data sets. The null hypothesis states that there is no difference between the RMSD values ( $\mu=\mu_{0}=$ $0)$, the alternative hypothesis is that the difference is greater than zero $\left(\mu>\mu_{0}\right)$, i.e. the second method is performing better than the first method. If the $p$-value is smaller than 0.05, the null hypothesis is discarded. Cases where the null hypothesis cannot be discarded are underlined.

\begin{tabular}{lll|rrrrr}
\hline Methods & Set & Measure & $n=1$ & $n=10$ & $n=25$ & $n=50$ & $n=100$ \\
\hline DG UFFopt-ETKDG & CSD & RMSD & $\underline{1.4 \cdot 10^{-1}}$ & $4.9 \cdot 10^{-4}$ & $3.9 \cdot 10^{-12}$ & $<2.2 \cdot 10^{-16}$ & $<2.2 \cdot 10^{-16}$ \\
& & TFD & $7.0 \cdot 10^{-4}$ & $5.0 \cdot 10^{-6}$ & $7.7 \cdot 10^{-5}$ & $1.6 \cdot 10^{-5}$ & $2.2 \cdot 10^{-7}$ \\
& PDB & RMSD & $\underline{0.34}$ & $\underline{0.46}$ & $\underline{0.83}$ & $\underline{0.99}$ & $\underline{0.78}$ \\
& & TFD & $\underline{0.11}$ & $\underline{0.46}$ & $\underline{0.70}$ & $\underline{0.98}$ & $\underline{0.48}$ \\
\hline DG & CSD & RMSD & $\underline{0.48}$ & $9.0 \cdot 10^{-9}$ & $<2.2 \cdot 10^{-16}$ & $<2.2 \cdot 10^{-16}$ & $<2.2 \cdot 10^{-16}$ \\
& & TFD & $9.7 \cdot 10^{-8}$ & $8.8 \cdot 10^{-12}$ & $2.1 \cdot 10^{-12}$ & $1.2 \cdot 10^{-14}$ & $<2.2 \cdot 10^{-16}$ \\
& PDB & RMSD & $\underline{0.87}$ & $\underline{0.61}$ & $\underline{0.59}$ & $\underline{0.48}$ & $\underline{0.51}$ \\
& & TFD & $\underline{8.7 \cdot 10^{-2}}$ & $\underline{5.9 \cdot 10^{-2}}$ & $\underline{0.26}$ & $\underline{0.47}$ & $\underline{0.88}$ \\
\hline
\end{tabular}

Table S4: $p$-values from one-sided paired $t$-tests of RMSD and TFD values of DG $(n=100)$ and ETKDG for different number of conformers, $n$, using the CSD and the PDB data sets. The null hypothesis states that there is no difference between the RMSD values ( $\mu=\mu_{0}=$ $0)$, the alternative hypothesis is that the difference is greater than zero $\left(\mu>\mu_{0}\right)$, i.e. the second method is performing better than the first method. If the $p$-value is smaller than 0.05, the null hypothesis is discarded. Cases where the null hypothesis cannot be discarded are underlined.

\begin{tabular}{lll|rrrrr}
\hline Methods & Set & Measure & $n=1$ & $n=10$ & $n=25$ & $n=50$ & $n=100$ \\
\hline DG $_{n=100}$-ETKDG & CSD & RMSD & $\underline{1.00}$ & $\underline{0.76}$ & $<2.2 \cdot 10^{-16}$ & $<2.2 \cdot 10^{-16}$ & $<2.2 \cdot 10^{-16}$ \\
& & TFD & $\underline{1.00}$ & $<2.2 \cdot 10^{-16}$ & $<2.2 \cdot 10^{-16}$ & $<2.2 \cdot 10^{-16}$ & $<2.2 \cdot 10^{-16}$ \\
& PDB & RMSD & $\underline{1.00}$ & $\underline{0.86}$ & $1.8 \cdot 10^{-8}$ & $<2.2 \cdot 10^{-16}$ & $<2.2 \cdot 10^{-16}$ \\
& & TFD & $\underline{1.00}$ & $5.5 \cdot 10^{-15}$ & $<2.2 \cdot 10^{-16}$ & $<2.2 \cdot 10^{-16}$ & $<2.2 \cdot 10^{-16}$ \\
\hline
\end{tabular}




\section{References}

(1) Schärfer, C.; Schulz-Gasch, T.; Ehrlich, H.-C.; Guba, W.; Rarey, M.; Stahl, M. Torsion Angle Preferences in Druglike Chemical Space: a Comprehensive Guide. J. Med. Chem. 2013, 56, 2016-2028. 